\title{
On Positive Eigenvalues of One-Body Schrödinger Operators*
}

\author{
BARRY SIMON \\ Princeton University
}

\section{Introduction}

The last twenty years have produced a rather extensive literature on the exact mathematical treatment of general features of the Schrödinger equation for one or many particles. One of the more intriguing questions concerns the presence of discrete eigenvalues of positive energy (that is square-integrable eigenfunctions with positive eigenvalues). There is a highly non-rigorous but physically appealing argument which assures us that such positive energy "bound states" cannot exist (c.f. [1], pages 30 and 51). On the other hand, there is an ancient, explicit example due to von Neumann and Wigner [2] which presents a fairly reasonable potential $V$, with $V(r) \rightarrow 0$ as $r \rightarrow \infty$, and which possesses an eigenfunction with $E=1$ (in units with $\hbar^{2} / 2 m=1$ ).

According to the excellent review article of Kato [3], Section 8, there are two general results which yield cases where $H=-\Delta+V(\mathbf{r})$ has no positive eigenvalues:

(a) In [4], Kato has proven that if $V=o(1 / r)$, then $H$ has no positive eigenvalue.

(b) In [5], Odeh has proven a similar result in case $V(\mathbf{r}) \rightarrow 0$ as $\mathbf{r} \rightarrow \infty$ and $\partial V / \partial r<0$ for sufficiently large $r$.

Both of the above statements require certain regularity conditions along with the indicated asymptotic behavior. For the case of spherically symmetric $V$, stronger results do exist (see e.g. [9]).

In this paper, we prove

THEOREM 1. Suppose $V$ is a real-valued function on $\mathbb{R}^{3}$ with the following properties:

(a) $V \in L^{2}\left(\mathbb{R}^{3}\right)+L^{\infty}\left(\mathbb{R}^{3}\right)$;

* The work for this paper was carried out at the Palmer Physical Laboratory, Princeton University, where the author is a Pre-Doctoral Fellow and was supported by the National Science Foundation. Reproduction in whole or in part is permitted for any purpose of the United States Government. 
$V=V_{1}+V_{2}$ with 1
(b) for some $R_{0}, V_{1}$ and $V_{2}$ are $C^{\infty}$ in $\left\{\mathbf{r} \mid r>R_{0}\right\}$,
(c) $\lim _{\mathbf{r} \rightarrow \infty} r V_{1}(\mathbf{r})=0$,
(d) $\lim _{\mathbf{r} \rightarrow \infty} V_{2}(\mathbf{r})=0$,
(e) $\lim _{\mathbf{r} \rightarrow \infty} \sup r \frac{\partial V_{2}}{\partial r}(\mathbf{r}) \equiv E_{0}<\infty$.

Then $-\Delta+V(\mathbf{r})$ has no eigenvalues in $\left(E_{0}, \infty\right)$.

We intend $\lim _{\mathbf{r} \rightarrow \infty}$ to be in the sense of $\infty$ having a neighborhood base of the exteriors of spheres, i.e., the limits are "uniform with respect to direction". We finally remark that this theorem clearly generalizes the results of Kato and Odeh.

\section{Proof of the Main Result}

The proof is unfortunately rather technical. We first prove a rather weak looking result which generalizes a result of Weidmann [6] (he deals with the many particle case; Theorem 2 is a generalization of his result when restricted to the one-particle case which is much simpler than the general case). An important input to the proof of the theorem is a series of technical lemmas from Kato [4] in a modified form. Finally, using a simple trick, we can turn the weak result of Theorem 2 into the stronger Theorem 1.

TheоReм 2. Let $V$ be a real valued function on $\mathbb{R}^{3}$ with the following properties:

(a) $V \in L^{2}+L^{\infty}$;

$V=V_{1}+V_{2}$ with

(b) for some $R_{0}, V_{1}$ and $V_{2}$ are $C^{\infty}$ in $M=\left\{\mathbf{r} \mid r>R_{0}\right\}$,

(c) $\lim _{r \rightarrow \infty} r V_{1}(\mathbf{r})=0$,

(d)' for $r>R_{0}, V_{2}(\mathbf{r})<0$,

(e) $)^{\prime}$ for $r>R_{0}, \frac{\partial V_{2}}{\partial r} \leqq-\frac{1}{r} V_{2}$.

Then $H=-\Delta+V(\mathbf{r})$ has no eigenvalues in $(0, \infty)$.

Proof: Suppose $H u=E u$ with $u \in L^{2}\left(\mathbb{R}^{3}\right)$ and $E>0$. Without loss of generality we may assume that $u$ is real-valued. By the Weyl lemma ([8], pg. 36),

1 The decomposition $V=V_{1}+V_{2}$ is unrelated to the decomposition assured by assumption (a). 
$u$ is $C^{\infty}$ on $M$. Define

$$
w(r, \theta, \phi)=r u(\mathbf{r})
$$

so that

$$
\left(-\frac{\partial^{2}}{\partial r^{2}}+\frac{1}{r^{2}} A_{\theta, \phi}+V(\mathbf{r})-E\right) w=0
$$

where $A_{\theta, \phi}$ is the Laplace-Beltrami operator on the sphere $S^{2}$ :

$$
A_{\theta, \phi}=-\left(\frac{1}{\sin \theta} \frac{\partial}{\partial \theta} \sin \theta \frac{\partial}{\partial \theta}+\frac{1}{\sin ^{2} \theta} \frac{\partial^{2}}{\partial \phi^{2}}\right)
$$

Following Kato [4], we regard $w$ as depending parametrically on $r$ by viewing $w(r,-)$ in $L^{2}\left(S^{2}\right)$. We remark that $A_{\theta, \phi}$ is a positive (unbounded) operator on $L^{2}\left(S^{2}\right)$. Following Odeh and Weidmann, we define for $r>R_{0}$

$$
F(r)=\left\|w^{\prime}\right\|^{2}-r^{-2}\left\langle w, A_{\theta, \phi} w\right\rangle+\left\langle w,\left(E-V_{2}(r)\right) w\right\rangle
$$

where $w^{\prime} \equiv \partial w / \partial r$ is regarded as lying in $L^{2}\left(S^{2}\right)$ and $\langle\rangle,\|\|$, represent the inner product and norm in $L^{2}\left(S^{2}\right)$. (We remark that $F$ has no direct physical interpretation; in particular it is not simply $T+V$ or $T-V$ ). By Kato's theorem [7] and hypothesis (a) of the theorem, $u$ and its first partial derivatives all lie in $L^{2}\left(\mathbb{R}^{3}\right)$ and $V_{2}$ is bounded in $M$. Thus,

$$
\int_{R_{0}}^{\infty} F(r) d r<\infty
$$

Since $F(r)$ is $C^{\infty}$ in $r$, we can evaluate, following Weidmann,

$$
\begin{aligned}
\frac{d}{d r}(r F(r))= & 2 r\left\langle w^{\prime}, w^{\prime \prime}-r^{-2} A_{\theta, \phi} w+\left(E-V_{2}(\mathbf{r})\right) w\right\rangle \\
& +\left\|w^{\prime}\right\|^{2}+r^{-2}\left\langle w, A_{\theta, \phi} w\right\rangle+E\|w\|^{2}+\left\langle w,-\frac{\partial}{\partial r}\left(r V_{2}\right) w\right\rangle \\
\geqq & \left\langle w^{\prime}, 2 r V_{1}(\mathbf{r}) w\right\rangle+\left\|w^{\prime}\right\|^{2}+E\|w\|^{2} .
\end{aligned}
$$

In the above we have used the equation of motion (1), the positivity of $A_{\boldsymbol{\theta}, \boldsymbol{\phi}}$ and the fact that $(e)^{\prime}$ can be rewritten as

$$
-\frac{1}{r} \frac{\partial}{\partial r}\left(r V_{2}\right) \geqq 0
$$


Since $\lim _{r \rightarrow \infty} r V_{1}=0$, we choose $X$ such that $|r V(\mathbf{r})|<k \equiv \sqrt{E}$ for $r>X$. Then, for $r>X^{\mathrm{r} \rightarrow \infty}$

so that

$$
\begin{aligned}
\left|\left\langle w^{\prime}, 2 r V_{1}(\mathbf{r}) w\right\rangle\right| & \leqq\left\|w^{\prime}\right\|\left|2 r V_{1}(\mathbf{r}) w\right| \\
& \leqq 2 k\left\|w^{\prime}\right\|\|w\|
\end{aligned}
$$

$$
\frac{d}{d r}(r F(r)) \geqq\left\|w^{\prime}\right\|^{2}+k^{2}\|w\|^{2}-2 k\left\|w^{\prime}\right\|\|w\| \geqq 0,
$$

that is

$$
\frac{d}{d r}(r F(r)) \geqq 0 \quad \text { if } \quad r>X
$$

We shall show below that, for some $r_{1}>X, F\left(r_{1}\right)>0$. Then (4) implies that, for $r>r_{1}$,

$$
r F(r)=r_{1} F\left(r_{1}\right)+\int_{r_{1}}^{r} d r \frac{d}{d r}(r F(r)) \geqq r_{1} F\left(r_{1}\right),
$$

so that

$$
F(r) \geqq \frac{r_{1}}{r} F\left(r_{1}\right)
$$

Since $F\left(r_{1}\right)>0$, we have $\int_{r_{1}}^{\infty} d r F(r)=\infty$, which contradicts $(3)$. Thus we have reduced the proof of Theorem 2 to the proof that $F\left(r_{1}\right)>0$ for some $r_{1}>X$. This is done using Kato's technical tricks in a series of lemmas (these parallel Kato [4], pages 409 410, but are included to make this paper self-contained). We define

$$
\begin{aligned}
F(m, t, r)=\left\|w_{m}^{\prime}\right\|^{2}+\left[k^{2}-2 k t r^{-1}\right. & \left.+m(m+1) r^{-2}\right]\left\|w_{m}\right\|^{2} \\
& -\left\langle w_{m}, V_{2}(\mathbf{r}) w_{m}\right\rangle-r^{-2}\left\langle w_{m}, A_{\theta, \phi} w_{m}\right\rangle,
\end{aligned}
$$

where $w_{m} \equiv r^{m} w$ and $k=\sqrt{E}$. Note that, for $m=t=0, F(m, t, r)=F(r)$.

Lemma 1. Suppose $0<t_{0}<k X$. Then there exists $m_{1} \geqq 0$ such that

$$
\frac{d}{d r}\left(r^{2} F\left(m, t_{0}, r\right)\right) \geqq 0 \quad \text { for } m \geqq m_{1} \quad \text { and } \quad r \geqq X \text {. }
$$

Proof: We first note that $w_{m}$ obeys the differential equation

(6) $\quad w_{m}^{\prime \prime}-2 m r^{-1} w_{m}^{\prime}+r^{-2}\left[m(m+1)-A_{\theta, \phi}\right] w_{m}+\left(k^{2}-V(r)\right) w_{m}=0$. 
We also have

$$
\begin{aligned}
& \frac{d}{d r}\left(r^{2} F\left(m, t_{0}, r\right)\right) \\
& =2 r^{2}\left\langle w_{m}^{\prime}, w_{m}^{\prime \prime}+\left(k^{2}-2 k t r^{-1}+m(m+1) r^{-2}-r^{-2} A_{\theta, \phi}-V_{2}(\mathbf{r})\right) w_{m}\right\rangle \\
& \quad+2 r\left\|w_{m}^{\prime}\right\|^{2}+\left(2 r k^{2}-2 k t_{0}\right)\left\|w_{m}\right\|^{2}-\left\langle w_{m}, \frac{\partial}{\partial r}\left(r^{2} V_{2}\right) w_{m}\right\rangle \\
& \geqq 2 r\left[(2 m+1)\left\|w_{m}^{\prime}\right\|^{2}+\left(k^{2}-\frac{k t_{0}}{r}\right)\left\|w_{m}\right\|^{2}+\left\langle w_{m}^{\prime},\left(r V_{1}(\mathbf{r})-2 k t_{0}\right) w_{m}\right\rangle\right] .
\end{aligned}
$$

In the above, (6) was used and the fact that $(\mathrm{d})^{\prime}$ and (e)' imply

$$
-\frac{\partial}{\partial r}\left(r^{2} V_{2}\right)=-r^{2} V_{2}-r \frac{\partial}{\partial r}\left(r V_{2}\right) \geqq 0
$$

Since $r>X$,

$$
\begin{aligned}
& \frac{d}{d r}\left(r^{2} F\left(m, t_{0}, r\right)\right) \geqq 2 r\left[(2 m+1)\left\|w_{m}^{\prime}\right\|^{2}+\left(k^{2}-\frac{k t_{0}}{X}\right)\left\|w_{m}\right\|^{2}\right. \\
&\left.-\left\|w_{m}^{\prime}\right\|\left\|w_{m}\right\|\left(k+2 k t_{0}\right)\right] .
\end{aligned}
$$

By taking $m$ sufficiently large we can insure that the right-hand side of this last equation is positive.

Lemma 2. There are constants $m_{0} \geqq 0$ and $R_{1} \geqq X$ such that

$$
F\left(m_{0}, t_{0}, r\right)>0 \quad \text { if } r \geqq R_{1} \text {. }
$$

Proof:

$$
\begin{aligned}
F(m, t, r)=r^{2 m}\left[\left\|w^{\prime}+m r^{-1} w\right\|^{2}+\left(k^{2}-2 k t r^{-1}\right.\right. & \left.+m(m+1) r^{-2}\right)\|w\|^{2} \\
& \left.-\left\langle w, V_{2}(r) w\right\rangle-r^{-2}\left\langle w, A_{\theta, \phi} w\right\rangle\right] .
\end{aligned}
$$

For some $R_{1},\left\|w\left(R_{1}\right)\right\| \neq 0$ (for otherwise $u$, the original eigenfunction, is 0 ). For $m_{0}$ sufficiently large, $F\left(m_{0}, t_{0}, R_{1}\right)>0$ and so, by Lemma $1, r^{2} F\left(m_{0}, t_{0}, r\right)$ $>0$ for all $r>R_{1}$.

LEMMA 3. For some $r_{1}>X, F\left(r_{1}\right) \equiv F\left(0,0, r_{1}\right)>0$.

Proof: Choose $R_{2}>R_{1}$ such that, for $r>R_{2}$,

$$
-2 k t_{0} r^{-1}+m_{0}\left(2 m_{0}+1\right) r^{-2}<0
$$


Since $\int_{R_{3}}^{\infty}\|w\|^{2}<\infty,\|w\|$ is not monotone increasing and so we can find $r_{1}>R_{2}$ such that

$$
\left.\frac{d}{d r}\|w\|^{2}\right|_{r=r_{1}} \equiv 2\left\langle w^{\prime}, w\right\rangle \leqq 0
$$

Then at $r=r_{1}$,

and so

$$
\left\|w^{\prime}+m r^{-1} w\right\|^{2} \leqq\left\|w^{\prime}\right\|^{2}+m^{2} r^{-2}\|w\|^{2}
$$

$$
\begin{aligned}
0\left\langle r^{-2 m_{0}} F\left(m_{0}, t_{0}, r_{1}\right) \leqq\right. & \left\|w^{\prime}\right\|^{2}+k^{2}\|w\|^{2}-\left\langle w, V_{\mathbf{2}}(\mathbf{r}) w\right\rangle-r^{-2}\left\langle A_{\theta, \phi} w, w\right\rangle \\
& +\left(-2 k t_{0} r^{-1}+m_{0}\left(2 m_{0}+1\right) r^{-2}\right)\|w\|^{2} \\
\leqq & F\left(0,0, r_{1}\right) .
\end{aligned}
$$

This completes the proof of Lemma 3 and thus also of Theorem 2. We are now ready to prove the main result.

Proof of Theorem 1: For each $\alpha>0$, let $V_{\alpha}(r)=V_{2}(r)-E_{0}-\alpha$. Then we can pick $R_{0}^{(\alpha)}>R_{0}$ such that, for $r>R_{0}^{(\alpha)}, V_{\alpha}(r)<0$ and $r \partial V_{\alpha} / \partial r+V_{\alpha}(r)<0$. Thus $V_{1}+V_{\alpha}$ obeys all the conditions of Theorem 2 and hence $H_{\alpha}=-\Delta+$ $V(r)-E_{0}-\alpha$ has no eigenvalues in $(0, \infty)$. Hence, for all $\alpha>0, H=-\Delta+V$ has no eigenvalues in $\left(E_{0}+\alpha, \infty\right)$ which proves Theorem 1 .

\section{Examples and Remarks}

A. Some typical potentials which have no eigenvalues in $(0, \infty)$ and which are not covered by any previous results are

$$
\pm \frac{z \sin r^{1 / 2}}{r^{2}} \text { and }-\frac{1}{r}+f(\mathbf{r})
$$

where $r f(\mathbf{r}) \rightarrow 0$ at $\infty$.

B. The potential

$$
V(r)=\frac{-32 \sin r\left[g(r)^{3} \cos r-3 g(r)^{2} \sin ^{3} r+g(r) \cos r+\sin ^{3} r\right]}{\left[1+g(r)^{2}\right]^{2}}
$$

with $g(r)=2 r-\sin 2 r$ is of particular interest. It is the original von NeumannWigner example (with their algebraic mistakes corrected) and has the eigenvalue +1 with eigenfunction

$$
u(r)=\frac{\sin r}{r\left(1+g(r)^{2}\right)} .
$$


For large $r$,

$$
V(r)=\frac{-8 \sin 2 r}{r}+o\left(\frac{1}{r^{2}}\right)
$$

so that $\lim \sup r\left(\partial V_{2} / \partial r\right)=16$. Thus for this example in which nasty things do in fact happen we can still say that there are no eigenvalues in $(16, \infty)$.

C. For the spherically symmetric $V$, a slightly weakened form of Theorem 1 follows easily ${ }^{2}$ from Satz 4.6 of [9]:

Proposition. If $V(r)$ is a function on $(0, \infty)$ which is differentiable and such that $V(r) \geqq \gamma>-\infty$ and $\lim \sup r|\partial V / \partial r|=E_{0}$, then $-\Delta+V$ has no eigenvalues in $\left(E_{0}, \infty\right)$.

Thus, in particular the conclusion of note $\mathbf{B}$ follows from previously existing theorems.

D. Since the existence of $E=0$ eigenfunctions is unrelated to the asymptotic behavior of $V$ (e.g. there are spherical square wells with $E=0, l \neq 0$, eigenfunctions $),{ }^{3}$ in general the conclusion $\left(E_{0}, \infty\right)$ of Theorem 1 cannot be replaced by $\left[E_{\mathbf{0}}, \infty\right)$.

E. The regularity conditions of Theorem 2 are slightly stronger than is necessary. The $C^{\infty}$ nature of $V$ was only needed to assure us that $u$ was actually a solution of the partial differential equation and that $u$ was $C^{2}$. For spherically symmetric potentials, this is implied by $V(r)$ continuous in $M$ and for the general case, where the Weyl lemma is needed, $V(\mathbf{r}) \in C^{3}$ in $M$ is sufficient (see e.g. [8], page 36 ; note that $C^{3}$ depends essentially on the fact that we are in $\mathbb{R}^{3}$ ).

F. All our results go through in $\mathbb{R}^{n}$ (except in $\mathbb{R}^{n}$, one needs $C^{m}$ with $m>\frac{1}{2} n+1$, rather than $C^{3}$ as the minimal smoothness necessary for $V(r)$ ).

G. In the case where $\lim V_{2}(\mathbf{r})$ does not exist, we can extend Theorem 1 slightly. The proof of Theorem 1 makes it clear that all we really need is

$$
\underset{|r| \rightarrow \infty}{\limsup } V_{2}(r) \leqq E_{0} \quad \text { and } \quad \limsup _{|r| \rightarrow \infty}\left(\frac{r \partial V_{2}}{\partial r}+V_{2}(r)\right) \leqq E_{0}
$$

for $H=-\Delta+V$ to have no discrete spectrum in $\left(E_{0}, \infty\right)$.

H. One can combine remarks $\mathbf{F}$ and $\mathbf{G}$ above to obtain some control over the many-particle problem; however, the condition $\lim \sup V(r)<\infty$ is quite

${ }^{2} \mathrm{~J}$. Weidmann, private communication.

${ }^{3} \mathrm{~V}$. Bargmann, private communication. 
strong for this case. For example consider $n$ equal mass particles with interparticle force $U$ and an infinitely heavy "nucleus" with particle nuclear force $W$, so that

$$
V=\sum_{i=1}^{n} W\left(r_{i}\right)+\sum_{i<j} U\left(\left|r_{i}-r_{j}\right|\right)
$$

Then $\lim \sup V(r)<\infty$ implies sup $U(r)<\infty-$ a condition which for forces like Yukawa and Coulomb forces is not satisfied.

Acknowledgements. The author would like to thank Profs. V. Bargmann, J. Weidmann and A. Wightman for their interest in this paper. I am indebted to the National Science Foundation for fellowship support during the course of this work.

Note added in proof: There is a minor technical flaw in the proof of Theorem 2 that may require a slightly strengthened smoothness condition. We have really only proven that $w \equiv 0$ outside a sufficiently large sphere. To conclude that $w \equiv 0$ everywhere, a unique continuation theorem is needed. For example, using the results of Müller (Comm. Pure Appl. Math. Vol. 7, 1954, pp. 505-516), one can prove $w \equiv 0$ when $V$ is $C^{2}$ and bounded on those compact sets which avoid a fixed finite set of singularities.

\section{Bibliography}

[1] Landau, L. D., and Lifshitz, E, Quantum Mechanics, Addison Wesley Publ. Co., Reading, Mass., 1958.

[2] von Neumann, J., and Wigner, E., Über merkwürdige diskrete Eigenwerte, Z. Physik, Vol. 30, 1929, pp. $465-467$.

[3] Kato, T., Some mathematical problems in quantum mechanics, Progr. Theoret. Phys. Suppl., Vol. 40, 1967, pp. 3-19.

[4] Kato, T., Growth properties of solutions of the reduced wave equation with a variable coefficient, Comm. Pure Appl. Math., Vol. 12, 1959, pp. 403-425.

[5] Odeh, F., Note on differential operators with purely continuous spectrum, Proc. Amer. Math. Soc., Vol. 16, 1965, pp. 363-366.

[6] Weidmann, J., On the continous spectrum of Schrödinger operators, Comm. Pure Appl. Math., Vol. 19,1966 , pp. 107-110.

[7] Kato, T., Fundamental properties of Hamiltonian operators of Schrödinger type, Trans. Amer. Math. Soc., Vol. 70, 1951, pp. 195-211.

[8] Fichera, G., Linear Elliptic Differeniial Systems and Eigenvalue Problems, Springer-Verlag, New York, Berlin, 1965.

[9] Weidmann, J., Zur Spektraltheorie von Sturm-Liouville-Operatoren, Math. Z., Vol. 98, 1967, pp. 268-302.

Received November, 1968. 